\title{
Perceptual and Sensory-Functional Consequences of Skin Care Products
}

\author{
Steve Guest $^{1}$, Francis McGlone ${ }^{2}$, Andrew Hopkinson ${ }^{3}$, Zachary A. Schendel ${ }^{4}$, Kevin Blot ${ }^{4}$, Greg Essick $^{1}$ \\ ${ }^{1}$ Regional Center for Neurosensory Disorders, School of Dentistry, University of North Carolina at Chapel Hill, Chapel Hill, USA; \\ ${ }^{2}$ School of Natural Sciences and Psychology, Liverpool John Moores University, Liverpool, UK; ${ }^{3}$ Unilever R \& D, Port Sunlight, \\ UK; ${ }^{4}$ Unilever R \& D, Trumbull, USA. \\ Email: steve_guest@dentistry.unc.edu
}

Received November $8^{\text {th }}, 2012$; revised December $10^{\text {th }}, 2012$; accepted December $19^{\text {th }}, 2012$

\begin{abstract}
Skin care products are often designed to provide tangible, physical benefits to skin health. Alleviation of dry skin and minimization of the signs of aging and post-injury scarring are important benefits targeted by many products on the market. Equally important to these benefits are favorable, desirable sensory attributes, without which products are unlikely to be used and repurchased. Other products are designed primarily to deliver sensory—or sensual—benefits (e.g., many cosmetic creams). This review considers the tactile sensory experience delivered by skin care products by examining: 1) their instrumentally-measured rheology and tribology; 2) their influence on the skin's mechanics (e.g., compliance); 3) their implications for changing sensory function (e.g., tactile sensitivity); and 4) the possibility that skin care products alter their own perception. Products that contain chemosensates (e.g., capsaicin, menthol) or pharmaceutical actives are not considered here. Although numerous perceptual-physical links have been reported, formulation rules by which products can be designed for optimal skinfeels are currently unavailable from the existing literature. This is because of inconsistencies among studies in the perceptual attributes investigated, the physical characterizations chosen to describe the products, and analysis methods employed. To provide a robust method for designing products with beneficial and desirable skinfeels, we propose the use of 1) a consistent lexicon that fully describes the perceptual experience of any product investigated, 2) a means of recording the mechanical events at the fingertip skin that occur when a skin care product is manually applied to the body. This approach contrasts with previous instrumental (in vitro) methods that may not generalize well to product-treated human skin (in vivo). Ongoing studies that record mechanical events at the skin surface show promise in identifying realistic models of the perception of skin care products.
\end{abstract}

Keywords: Review; Rheology; Tribology; Perception; Sensory Function; Tactile

\section{Introduction}

Skin care products are generally designed to provide protective or reparative benefits, such as in maintaining skin moisture [1], preserving skin barrier function [2], reducing the prominence of scar tissue [3] or lessening the signs of skin aging [4]. Equally important to these benefits are the perceptual attributes of products, as these contribute substantially to whether a product is liked and thus used. For example, if a skin cream is perceived as unpleasant to touch, it is unlikely to be used voluntarily even if it is potentially beneficial to skin health ${ }^{1}$. Additionally, some skin care products are designed such that their primary benefit is perceptual, such as in conveying soothing or comforting feelings [5], as opposed to tangible, benefits to the skin.

The perception of skin care products, like other tactile stimuli, is dependent on mechanoreceptors in the skin, the outputs of which result from shear or indentation [6].
The physical properties of products (e.g., viscosity of fluids or surface texture of solid materials), in combination with touch behavior, determine the time-varying pattern of mechanical stimulation of the skin surface. However, skin care products differ from most other tactile stimuli in that they can actually alter the physical properties of the skin, such as by changing its state of hydration [7]. Such changes can alter the pattern of mechanical stimulation of the skin surface produced by touch. That is, as well as having direct tactile perceptual characteristics, products can alter sensory function. Fur-

\footnotetext{
${ }^{1}$ Although desirable, pleasant skinfeel would generally be the target of a skin care formulation, sensations usually considered unpleasant can in some circumstances be intended. Such sensations can convey a sense that the product is providing a functional benefit. The astringent oral sensation produced by mouthwash is an example. This has no consequences for this review; where the physical basis of pleasant benefits is mentioned, this clearly includes the possibility of engineering a lack of such benefits.
} 
ther, these changes in sensory function can affect the subsequent perception of the skin care product as well as the perception of tactile stimulus objects.

This review highlights how the perception of skin care products is linked to the physical properties of those products, to changes in skin mechanics the products cause, and to sensory-functional changes that follow from the changes in skin mechanics. It is important to note that non-cutaneous inputs are also important in product perception, such as product appearance and packaging. Such multisensory influences will not be considered here, but examples of multisensory relevance in consumer product perception are readily available $[8,9]$.

\section{Perceptual Dimensions and Skin Care Products}

A necessary stage in understanding how skin care products are perceived and alter perception of the skin is to know what percepts are important to study, i.e., what percepts skin care products can potentially convey or alter. Typically, the perceptual attributes that have been used in studying consumer behavior have been chosen in a fairly ad-hoc manner, based presumably on introspection and practical experience. Thus we commonly see ratings made, for example, of tactile percepts of smoothness [5,10] and softness [10-12] and the visual percept of gloss $[11,12]$, but little consistency among studies in terms of what is - or should be - rated.

Providing a more principled way of assessing perception is an important issue for perceptual research in general, and attempts have been made to determine what independent dimensions describe how our perceptual worlds are represented. For the tactile perception of nonfluid materials, multidimensional scaling (MDS) methods have established that the primary dimension of touch perception is Rough-Smooth [13-17]. A secondary dimension is Hard-Soft, with higher dimensions less clear, but perhaps including Springy-Inelastic [13] or StickySlippery. These studies assist researchers in consumer science pertaining to non-fluid materials by suggesting what percepts are important and should thus be studied [15].

The important tactual dimensions of fluid-covered surfaces are less well known than those for dry materials. Some relevant studies exist, although most suffer from shortcomings that hinder their interpretation and general usefulness. For example, Almeida and colleagues [18] obtained subjective ratings of seven diverse formulations (emulsions, lanolin/petrolatum, a hydrogel and oleogels) from 10 naïve panelists. Ratings were made for selections of attributes related to appearance, pickup, rub-out, afterfeel and longer-term afterfeel. Principal components analysis (PCA) suggested that three components described the attribute relationships well. The primary com- ponent described a combination of pickup attributes, residue, stickiness and gloss after application. The nature of this component illustrates the main issue with this and similar studies, namely the component structure did not summarize the ratings in an easily interpreted manner. There are two main reasons for this: first, the inclusion of tactile, visual and temporal attributes leads to complex components. In this case, it is unclear how one would use the component structure reported by Almeida et al. to decide what perceptual attributes are important in skin cream perception. Second, the attributes initially rated were not chosen such that they included all likely applicable percepts. Similar issues are present in other relevant studies [11,19].

Recently, we [20] characterized the tactile perception of stimuli in a more general manner, with the underlying goal of providing a lexicon of the words that are necessary to describe the experience of touch for dry and - in a subsequent study-lubricated surfaces [21]. The work built upon methods used in the development of standard clinical tools, primarily the McGill Pain Questionnaire [22], and a more recent methodology describing the development of a texture description lexicon [23]. The latter began with a comprehensive set of words, obtained from a dictionary search, which could be used to describe the tactile experience of any conceivable texture. Similarity judgments of all pairings of the more common words were then used to form a dissimilarity matrix, describing the perceptual distances among all words. Finally, MDS was used to map these distances along orthogonal axes. The MDS solution suggested the psychological constructs the words described, and where in semantic-perceptual space each word fell. The relative locations of words allow one to determine which are similar enough that one or more might be omitted from any texture description lexicon.

We [20] used a similar approach to develop the "Tactile Perception Task" (TPT), a set of attributes designed to enable ratings of all of the important tactile perceptual attributes, but with some important differences. First, we recognized that tactile perception can be divided into its sensory/discriminative and emotional attributes (after [22]), supported by recent research identifying a system of peripheral nerves in the skin that code for the pleasant properties of touch [24-26]. Sensory attributes are those that refer to the pure sensations that occur when experiencing touch, such as terms describing roughness and smoothness. Emotional attributes describe the feelings that occur when touching or being touched, such as terms describing comfort or sensuality. On this basis, separate sensory and emotional word spaces were produced. Second, we considered words that describe the sense of touch in general, not just texture perception. Finally, we extended the work to consider the specific case of skin 
care product perception, i.e., fluids [21].

Our suggestion was that words describing the overall sensory experience of touch (i.e., semantic-perceptual space) fall along three orthogonal axes, approximating Rough-Smooth, Dry-Wet and Hot-Cold (Figure 1), but when actual dry, textured stimuli were assessed using these words (i.e., using the TPT), orthogonal factors emerged describing, in decreasing order of importance, Roughness, Slip, Firmness and Pile. The subsequent extension of this work to the perception of skin care products [21] suggested five orthogonal sensory factors approximating Wetness, Texture, Slickness, Silkiness and Viscosity. Water is, of course, a fluid that is of paramount importance for humans, and the primacy of the wetness factor may reflect water's critical role in our lives. Later in this review we specifically address what it is that may convey the feeling of water against the skin.

Comparison of the factors emerging from the two stimulus types considered by Guest and colleagues (i.e., dry textiles versus fluids) suggests that the sensory-perceptual structure of fluid perception is quite different to that of dry surface perception. This indicates that referring to the work on dry textures [13-17] when determining what percepts should be studied when assessing skin care products is unlikely to be optimal; the important percepts differ between dry and lubricated surfaces.

In contrast to sensory words, those describing the emotional experience of touch fell into three factors, namely Comfort, Arousal and (tentatively) Sensory Quality. The first two emotional factors matched very well those from prior work in social psychology, which suggested that any emotional experience is embodied with certain amounts of three independent qualities, namely Pleasure, Arousal and Dominance [27-30]. Unlike the sensory experience of touch, the factors describing the emotional experience of touch appear quite similar for the tactile experience of dry and fluid stimuli [20,21]. The emotional experience of Dominance has not emerged in these studies, perhaps because Dominance is only of consequence in interactions between, or assessments of, other humans (perception of facial expression, [31], perception of body posture, [32]). In contrast, it seems likely that Pleasure (or Comfort) and Arousal are indeed universal dimensions of any emotionally based judgment.

These findings suggest that adaptation of consistent attributes for the study of skin care products would greatly assist in synthesizing different studies, and in assessing all of the tactile attributes critical to the perception of skin care products. The TPT is one possible set of such attributes. The level of detail available via the TPT, and its division of perception into sensory and emotional components has allowed distinctions to be found between body sites in their response to tactile stimulation, consis-
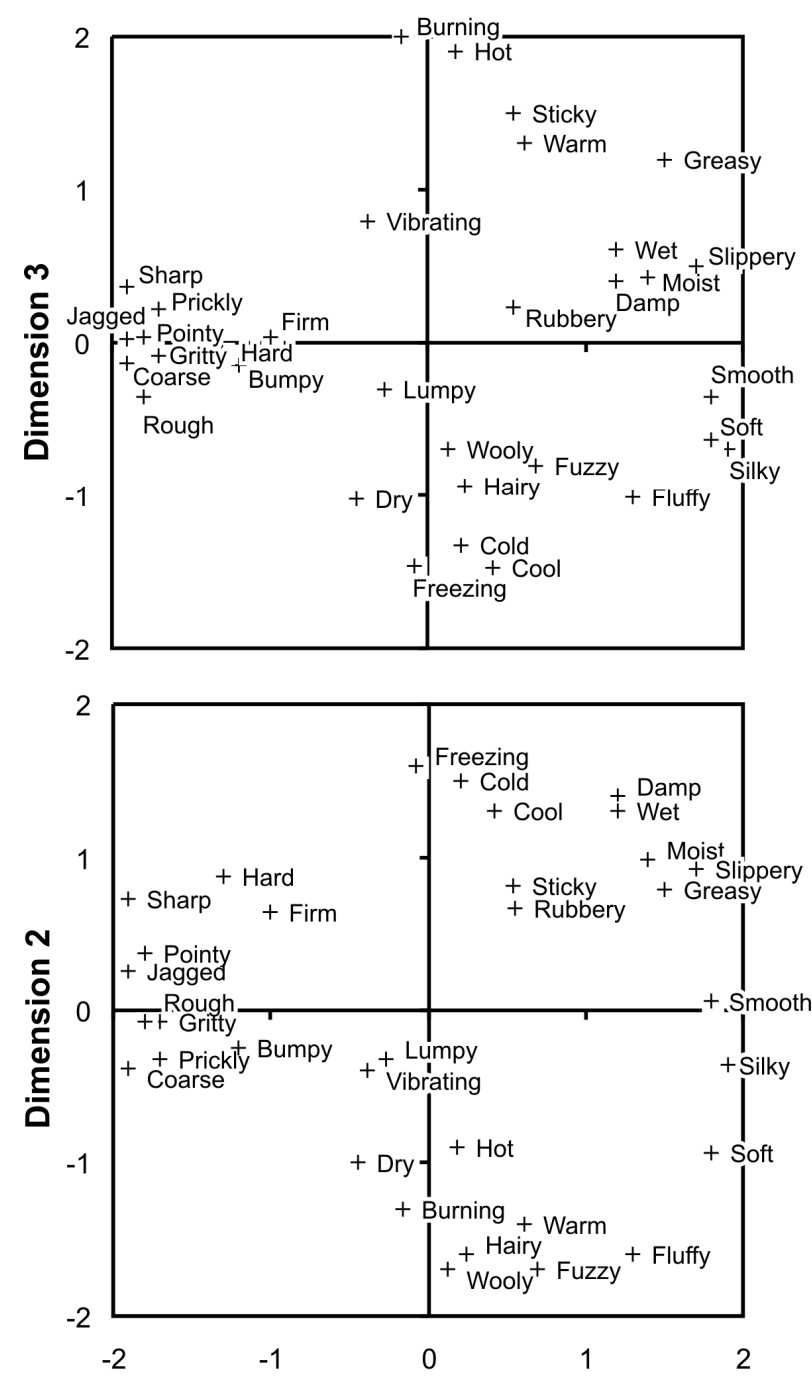

Dimension 1

Figure 1. The locations in semantic-perceptual space of words describing the sensory experience of touch.

tent with underlying differences in skin innervation [33] as well as the differences in skin care product perception mentioned earlier [21]. These results suggest the TPT may be a useful tool for further analysis of skin care product perception.

\section{Relationships between Perception and Physical Characteristics of Skin Care Products}

In order to optimize the perceptual attributes of skin care products, one needs to know how percepts are linked to measureable physical characteristics of fluids. With this knowledge, it is then possible to potentially engineer fluids with desired perceptual characteristics. A convenient distinction may be made between rheological and tribological aspects of fluids. The former pertains to bulk 
flow properties of fluids, the latter to the lubricating effects of fluids when present in a thin layer between sliding surfaces.

\subsection{Viscosity and Other Bulk Rheological Parameters}

The field of research in which the relationships between perceptual characteristics and rheological properties of fluids is studied is often referred to as Psychorheology, the sensory judgment of rheological properties, and is a well-studied area within foods research [34-38] but more rarely studied in the skin care area [39]. Viscosity is perhaps the most popular bulk rheological measure considered in tactile perception work. Individuals can assess relative viscosity levels quite well, using haptic and visual means [40], and changes in viscosity are associated with distinct percepts. For example, single measures of viscosity for eight emollients have been shown to correlate positively with ratings of stickiness, and negatively with soft and slippery percepts [11].

However, single measures of viscosity are not necessarily good targets for perceptual-physical links because for many fluids viscosity is not constant for all conceivable touch motions. For example, the apparent viscosity of non-Newtonian fluids depends upon the shear rate. For the assessment of skin care products, this translates as changes in viscosity according to how quickly an exploring finger moves over a surface treated with a skin care product.

Prior work in the oral perception of foods highlights the importance of considering these nonlinearities in viscosity. For example, it has been found that ratings of fluid viscosity correlate best with instrumental measures of viscosity at specific shear rates or stresses [35,36], where the applicable shear rate or stress depends on the fluid viscosity. Presumably these rates and stresses are developed in the mouth according to the manipulation of the fluid over the oral tissues. Similar findings have been reported for the haptic or proprioceptive assessment of fluid viscosity [41].

Some studies of relevance to skin cream perception have characterized viscosity changes according to shear rate, and product thixotropy (i.e., hysteresis in shear stress vs. shear rate profiles according to changes in shear rate, or over time at constant shear rate), but nevertheless have still used a single point estimate of viscosity to relate to extra-oral tactile perception, i.e., the relationships between viscosity and shear rate were ignored [12]. In this particular case, the perception of oil-in-water emulsions was but weakly related to viscosity and thixotropy, the strongest relationships being positive between hardness and allied percepts and viscosity. Viscosity and thixotropy were correlated in the sample such that perceptual relationships were essentially the same with each physical measure.

More sophisticated measures than single point estimates of viscosity have been considered, such as the shear stress at which a product's maximum viscosity occurs, and the viscosity maximum per se [42]. Unfortunately the perceptual rating collected in this case was limited to a four-category, broad-based judgment of "acceptability" which does not let one know what distinct percepts might have been driven by the shear stress - viscosity relationships.

A brute force method of finding perceptual-rheological links has been reported for the perception of creaminess in foods (custards: [43]). Eighty-four rheological parameters were measured for 47 custards, and partial least squares regression used to find latent variables relating sets of these parameters to ratings of creaminess. Despite this rather comprehensive approach, just three rheological parameters, those describing fluid behavior during a dynamic stress-sweep (oscillatory test with increasing stress amplitude), predicted creaminess perception quite well (loss modulus G" at $33 \mathrm{~Pa}$, and two critical strain measures; square of correlation coefficient c. 0.43). In simple terms, creamy custards were initially stiff, but flowed easily with minimal deformation.

Two similar approaches have been reported that relate more to skin care products. In the first a set of 29 branded skin care products and model fluids was studied by Wegener [39]. A wide range of rheological parameters were measured, with findings including positive relationships between sensory tackiness and stickiness and viscosity measured at high shear rates $\left(10,000 \mathrm{~s}^{-1}\right)$, and negative relationships between sensory spreadability and oiliness and viscosity. Wegener also found that wetness was negatively correlated with viscosity measured at very low shear rates $\left(0.001 \mathrm{~s}^{-1}\right)$ and to several additional parameters measured by oscillatory rheology. A more recent study of 40 basic skin creams [44] found that firmness, thickness and spreadability could be predicted from oscillatory rheology parameters measured at large strain amplitudes.

\subsection{Friction and Other Tribological Factors}

A great many factors can influence the frictional characteristics of skin when it is not treated with a skin care product. For example, the presence of natural sebum, the instrumentally-defined roughness of the surface contacting the skin, and the body site are all important. These and other factors have been reviewed recently $[45,46]$. Regarding the friction specifically of treated skin, the presence of a product can alter the friction coefficient of touch involving that treated skin as a consequence of hydration of the stratum corneum, which increases the friction coefficient of the skin surface when it contacts a wide variety of surfaces (textiles: [47,48-50]). Further, 
and perhaps more importantly, the presence of a film of product per se has its own frictional characteristics. A complication regarding the friction of treated skin is that the thickness of the fluid film on the skin significantly alters the relative influence of the product versus the skin's characteristics. Lubrication can occur as "boundary", "mixed" or "hydrodynamic" regimes as film thickness increases, with each of these regimes having different lubrication characteristics (Figure 2). For example, one may contrast boundary lubrication, where the texture of the skin plays a role in both the friction between the treated skin and exploring fingertip and the vibration that is generated, and hydrodynamic lubrication where the skin's texture is likely to be inconsequential. These regimes are known to influence the perception of foods in the mouth, and are presumably just as important in the extra-oral perception of fluids (see [51] for a review).

The initial feel of a product on the skin is likely to be dominated by the product's characteristics, rather than the skin's characteristics, at least assuming the initial layer of product is thick enough to provide hydrodynamic lubrication. This has been empirically shown for the specific case of suitably thick films of Newtonian fluid; the underlying roughness of involved surfaces did not influence the friction coefficient [52].

Indeed, the presence on the skin of products such as petrolatum, glycerin or mineral oil initially decrease the friction coefficient and these products are typically perceived as initially greasy, with greasiness ratings correlated strongly with the initial reduction in the friction coefficient [50]. Subsequently the friction coefficient increases over a period of hours, and perceived greasiness decreases. The friction coefficient changes could occur on the basis of increasing skin hydration as the product acts as a barrier to evaporative water loss through the skin, and on the basis of changes in the product as constituents are absorbed into the skin, or migrate from the initial application site. It is unclear what lubrication

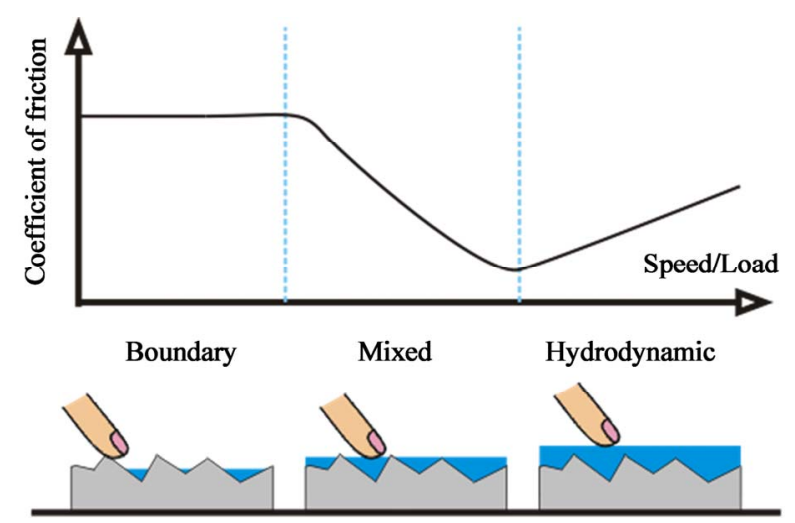

Figure 2. Lubrication regimes that may occur during touch of surfaces with a fluid layer interspersed between exploring finger and body site or other surface. regime was present at the extended time lags from initial application, and therefore it is unclear to what extent changing skin friction versus changing product mechanics were driving changes in the greasiness percept.

Although basic frictional measures such as those described above are easy to obtain by applying the applicable instrument to live skin, such measures do not take into account that friction is unlikely to remain constant even during short-term assessments of skin care products. One reason for this is that friction can vary as touch speeds and loads vary; indeed, friction depends on viscosity, which is dependent on touch speed and load (Figure 3). For example, for non-Newtonian fluids such as polysaccharide solutions, the speed and load with which they are explored alters their friction coefficient [53]. Further, it is known that the touch speeds and loads chosen indeed vary during natural tactile behaviors [54]. These observations suggest that for most skin care products a single measure of friction will not necessarily be representative of any putative friction-perception links. In fact, the perception of dry materials is also influenced by touch speed and load (see [55] for a review), although the basis of this is more likely to reside in the perceiver, than in changing mechanics at the skin-surface interface. This is so because the mechanics of most dry materials will not exhibit the dramatically changing frictional properties that fluid coated surfaces can.

Although frictional changes are clearly important in product perception, the perceptual significance of such changes is not simple, or consistent among body surfaces. For example, damaged hair has an increased friction coefficient, and treatment of damaged hair with conditioner reduces the coefficient such that it is comparable to that of undamaged hair [56]. Thus the reduction in friction coefficient in damaged hair suggests a desirable benefit, namely reduced hair damage. In contrast, a reduction in

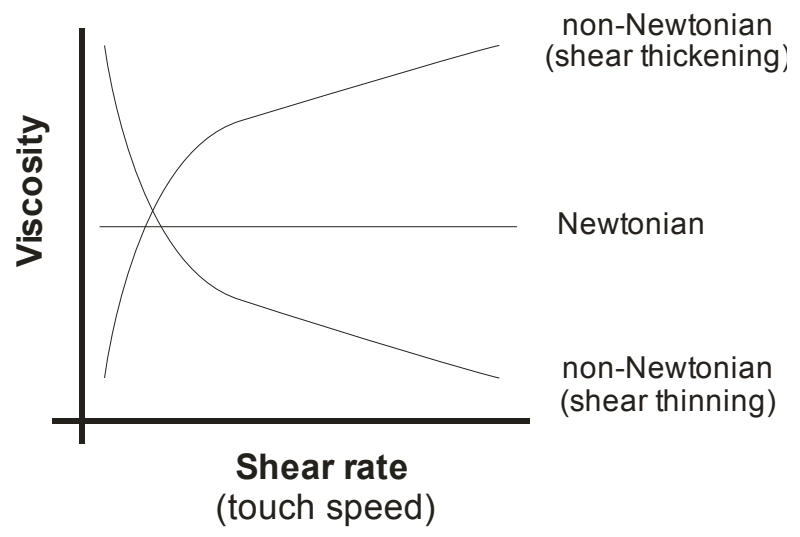

Figure 3. Fluids can vary in apparent viscosity according to how quickly they are undergoing shear; in the case of tactile exploration of lubricated surfaces, shear rate is effectively the speed of motion of the exploring finger against the surface. 
the friction coefficient of undamaged skin does not signify a positive benefit per se, because desirably hydrated skin actually has relatively high friction. Further, and as noted earlier, skin care products that reduce friction can have associated unpleasant feelings of greasiness [50].

\subsection{Instrumental Measurements from Live and Simulated Touch}

The studies summarized above have used conventional instrumental measures of viscosity, friction and other physical attributes of products in order to seek perceptual-physical links. An alternative approach would be to gather instrumental measures during the actual touch made by an individual against a body site while applying or assessing a product. This approach has the great advantage that it could record the mechanical events at the fingertip (or other body surface) that stimulate the mechanoreceptors during touch. Mechanical events include instantaneous frictional forces, but also vibrations normal and tangential to the point of contact. Mechanoceptor activity is of greater potential interest than conventional instrumental measures in forging perceptual links because it is the mechanoreceptor outputs that predominately determine tactile percepts. However there is currently no technique that allows concurrent recording of friction during skin-skin contact, i.e., intra- or interpersonal touch [10], although vibration stimuli have been measured indirectly (a short distance away from the contact) using microphones, hydrophones and accelerometers [57].

Although it is not currently feasible to concurrently record frictional forces during naturalistic touch between two body sites, devices exist that allow concurrent recordings for explorations made by the finger against a (treated) surface. Several such devices have been reported [58-61]. Each essentially consists of a rigid plate attached to sensitive force transducers, allowing real-time recordings of touch-relevant mechanical events that occur at the point of contact between the plate and exploring fingertip. The substrate over which products are explored can be a suitable skin analog.

For the most part, the perception of lubricated surfaces has not been the focus of work involving these "force plate" devices. However, using such a device we recently investigated the role of friction in the perception of a diverse range of 15 fluids which included some skin care treatments [61]. Each of these fluids was explored using a wide range of touch speeds and loads. Each fluid was separately rated using a slightly modified version of the TPT. Fourteen mechanical events were considered, consisting of vibrations normal and tangential to the fingertip, and friction-related parameters. The friction parameters described the relationship between friction and touch speed and load, as well as the friction forces at selected touch speeds and loads. Factor analysis was used to separately summarize the mechanical events, and the sensory and emotional attribute ratings. A friction factor was the second most important extracted that differentiated the fluids, encompassing $28 \%$ of the variance in mechanical events. Greater perceived fluid viscosity was correlated with greater scores for a composite friction factor, and smaller scores for two composite vibration factors. The inverse relationships were seen for perceived fluid wateriness. Unlike the work of Nacht et al., there was no indication that friction and perceived greasiness were strongly related, although similar products were perceived as greasy in the two studies (e.g., different petrolatum-based products). It is possible that this lack of relationship was mediated by the use of factors to summarize attributes in the Guest et al. study; greasy was not analyzed separately from a factor that included several other attributes.

In a separate study [44] but using the same force plate, the friction and vibration properties of 12 basic skin creams were measured at different times over 10 minutes as the creams dried on a skin proxy. It was found that firmness and thickness (which pertain to the early stages of cream application to the skin) could be predicted from the friction coefficient measured early on in the $10 \mathrm{~min}$ utes and (paradoxically) from a load dependence of the friction coefficient late in the 10 minutes. It was also found that drying and final greasiness (sensory percepts related to the feel of the skin after the cream has been rubbed in) could be predicted from the load dependence of the friction coefficient in later episode. These latter percepts were not well predicted by rheology, understandably so because the physical properties of a dried cream are not likely to be well characterized by the bulk rheology of the fresh cream.

As yet unpublished data we have collected via a similar force plate, but using an even larger set of products and model fluids (50 items), suggests that correlations between the coefficient of friction and perception are maximized at particular touch speeds and loads, the value of which depend on the percept being rated. For example, oiliness appears associated with low friction, specifically at low touch speeds, and high touch loads. In contrast, thick or firm percepts appear associated with greater friction specifically at high touch speeds, and low touch loads.

Instead of investigating an active participant assessing a product against a substrate, an alternative approach is to artificially simulate a finger rubbing action and to measure the frictional-and other-phenomena that occur during such rubbing $[62,63]$. In effect these are "artificial finger" devices. The tribometer reported in the 2009 study [63] was used to measure the temporal evolution of friction which was then characterized by seven 
parameters such as the static friction coefficient, kinetic friction coefficient and amplitudes of vibration. Measurements were made on 10 product formulations, all consisting of mica and acrylic particles in oil. Each product was rated in terms of smoothness, silky feeling, velvety feeling, softness and skin-adhesion ability (i.e., stickiness). Unfortunately, ratings of the sample set were restricted to ratings on a five point scale, made by three experienced observers. The ratings suggested that only softness and skin-adhesion ability varied widely among the products (see Figure 8 in Horiuchi et al.). Those two percepts were well fitted by multiple linear regressions using the tribological parameters as predictors $\left(\mathrm{R}^{2}=0.70\right.$ and 0.81 , respectively).

A modification of the same tribometer has also been used to generate inputs (11 instrumental inputs) to an artificial neural network designed to predict the same five perceptual quantities [64]. In this case, inspection of the network weights showed quite different contributions of the 11 inputs for each of the five percepts. The contribution patterns did not suggest easily interpretable relationships between mechanical events and percepts, although some percepts were more simply linked to mechanical events than others. For example, greater smoothness was associated with low dynamic friction and a relatively quick stabilization of dynamic friction in the sliding apparatus. In the most recent variant on these studies [62] relative displacements of a fixed versus movable plate with one of seven interspersed product were considered, as the movable plate travelled across the fixed plate. These displacement-time relationships would be analogous to variations in the frictional force with position. It was proposed that temporal patterns of relative displacement could be the basis on which the different products were perceived as different. Temporal changes in the stimulus delivered to the fingertip have been suggested as important in liquid-relevant percepts, such as the perception of water, which is addressed later in this review.

These artificial finger-based studies had the clear goal of a major aim of skin care perception work, namely that of obtaining good predictions of human responses to skin creams using instrumental measures only. A major issue with all of the devices reported to date is that they are very limited in the degree to which they truly replicate the physical stimulus that occurs during skin-skin contact, i.e., that present during actual assessment of skin care products by consumers. As such, the mechanical events generated and analyzed may be inadequate descriptors of the actual stimulus received by the skin's mechanoreceptors. Other groups have sought to better replicate the physics of the human finger pad [65] and its spatial tactile sensitivity [66]. Both of these studies were concerned with only dry sample surfaces, but better consideration of finger biophysics is likely to be fruitful in designing better artificial finger devices for perceptual-physical analysis of lubricated surfaces.

A different approach to modeling perceptual responses from a complex of instrumental measures has been reported as Integrated Sensory Response Modeling (ISRM: $[67,68])$. This methodology has been developed and proposed to capture and mathematically integrate different, independent phenomena which are hypothesised to be all involved in quantitatively modifying the quantitative expression of one perceptual response (e.g., the saltiness of tomato soup or the thickness of a skin cream). ISRM has been developed using intensity differences measured for sensory attributes of consumer goods which generate both many variations in time dependent availability or release of perceptual stimuli as well as many multi-modal perceptual interactions. ISR-models integrate mathematical constructs of instrumental and compositional measures representing time dependent/release availability of perceptual stimuli with mathematical constructs of multimodal sensory interactions, aiming to produce the best possible fit while avoiding overfitting. In effect, an ISRmodel consists of a nonlinear regression linking a perceptual attribute to many physical stimulus attributes, which may be derived from conventional instruments, or potentially from mechanical events recorded during live finger tracking, or from the use of artificial finger devices.

Although the results from artificial finger devices and the use of techniques such as ISR-modeling might allow instrumental predictions of perceptual quantities, any underlying perceptual mechanisms are quite distant. For example, it is unclear how the maximal resonance amplitude measured for sliding contact between two plates is related to receptor activity in the skin during skin-skin touch. There is a risk of obtaining misleading or spurious correlations, especially when many physical and perceptual quantities are considered in a single study.

\subsection{The Specific Case of Water/Wetness}

Water alone is not typically considered a skin care product, but it is the most important fluid of all to humans. Further, water is a common base ingredient in product formulations. Distinct water receptors do not exist in the skin and therefore the perception of the presence of water must occur due to specific patterns of mechanical stimulation of the skin, likely in combination with other effects such as local cooling or warming. The underlying physical stimulus that leads to the perception of water or wetness is of relevance to skin care products because one might wish to alter the feeling of wetness a product elicits. For example, one might want to enhance the perception of wetness, perhaps in order to enhance feelings of refreshment, or reduce the perception of wetness, which 
is undesirable for some skin care products (e.g., antiperspirants).

Humans are quite sensitive to water as a stimulus, and can judge the relative wetness of soaked fabric swatches similarly well across body sites [69], and among different wetted materials [70]. In the former study Ackerley and colleagues found that the sensitivity to wetness differences was very similar among sites that varied greatly in mechanoreceptor innervation density. Thus, it seemed that the perception of water or wetness was not carried by a spatial receptor code. Instead, the researchers hypothesized that temperature changes in the skin due to evaporative cooling were the basis on which wetness levels were judged. Early studies have suggested that in some contexts, cold stimuli are perceived as wet [71-73], a finding that has been replicated more recently for the oral perception of fluids [74]. These studies generally support the cooling hypothesis. As such one would at first glance expect most products to initially produce an enhanced sensation of wetness as any volatile compounds evaporate (i.e., evaporative cooling), or simply because an initially cool product warms up to skin temperature by absorbing heat from the skin. However, although such physical cooling has been observed and quantified [75], along with the expected cooling sensations, a paradoxical sensation of warmth has been reported after treatment with petrolatum [76]. The basis of this sensation could be a reduction in trans-epidermal water loss (TEWL) due to petrolatum's barrier function, i.e., reduced evaporative cooling. Regardless of the underlying physical mechanism, this finding suggests that the perception of the wetness of petrolatum against the skin might be lowered, due to the paradoxical warmth. Cooling-based enhancement of wetness against the skin must take into account trans-epidermal water loss and evaporation of any volatiles in the applied product.

Although thermal effects appear important in signaling wetness, there is a body of evidence that suggests particular temporal patterns of mechanical stimulation engender a feeling of water against the skin. When rubbing a water-lubricated glass surface, the finger tends to move with a characteristic stick-slip motion [77,78]. Silicone oil has been show to feel more similar to water as its stick-slip characteristics under exploration approach those of water. The characteristics of the silicone oil were manipulated by changing the texture of the substrate over which it was explored [77]. The importance of these temporal-mechanical effects in perception is highlighted by the finding that the discrimination of the degree of wetting of textile samples is much better for dynamic, versus static, touch [70].

In sum, the results of these water-centered studies suggest that one could enhance feelings of wetness in skin care products by attempting to match the stick-slip behavior of water, along with including the potential for relatively large evaporative cooling by inclusion of volatiles. Or, placing this in counterpoint, skin care products that exhibit water-like stick-slip behavior when touched, and which cool the skin substantially are likely to be perceived as wet or watery.

\section{The Influence of Skin Care Products on Skin Mechanics and Sensory Function}

Thus far, this review has considered links between the physical properties of skin care products and how they are perceived. The remainder of the review will consider how skin care products can change the mechanical properties of the skin itself. This is an important topic because an important consequence of a product changing the skin's mechanical properties is that sensory function might be altered, i.e., the sensitivity of the perceiver to touch of various types. If sensory-functional changes occur as a consequence of the use of skin care products, the perceiver's response to subsequent stimuli could be altered. For example, the texture of clothing in contact with the body might be experienced differently when the skin is treated versus when it is in its untreated state. Product physical properties and skin mechanics cannot be perceptually disentangled.

Skin care products can readily increase skin hydration $[1,7]$. The most obvious change in skin mechanics that might be caused by hydration is increased softness or decreased stiffness [79], although suggestions have been made that the effect of hydration on skin stiffness is subject-dependent [80]. Of relevance is that inter-individual variations in untreated finger skin hardness have been related to tactile sensitivity, with pressure perception thresholds being greater in individuals with harder skin [81]. This suggests that increasing skin compliance via hydration might well increase some measures of tactile sensitivity.

This possibility was addressed by Lévêque and colleagues' task [82], who found that a $5 \%$ glycerol moisturizer significantly increased the moisture content of the skin of aged individuals (mean age 66.5 years), and increased spatial acuity at the cheek as measured by a twopoint discrimination. Separate microneurography of sites on the lower limb indicated that fast- and slow-adapting skin mechanoreceptor activity decreased with increased skin hydration. The researchers argued that spatial acuity was improved with hydrated, compliant skin because the two prongs of the stimulator provided more discrete stimulation than with less hydrated, and thus less compliant, skin (Figure 4).

Following from Lévêque et al.'s work, one may propose that the effect of skin hydration on the perception of a mechanical stimulus should depend on the nature of the 
(a)

(b)

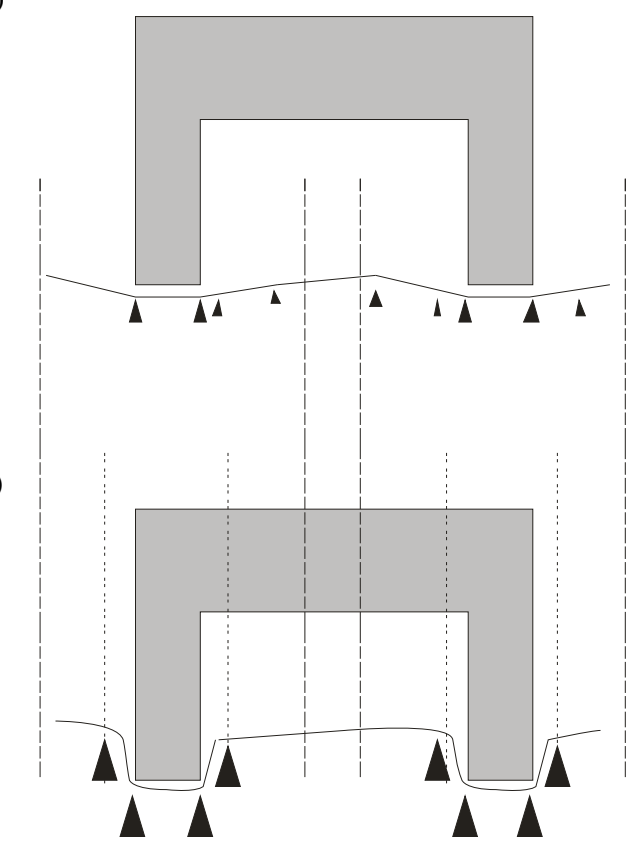

Figure 4. Patterns of strain in (a) low versus (b) high compliance skin, as hypothesized by Leveque et al. (2000) to occur in low versus high stratum corneum hydration in response to a two-pronged stimulator. These patterns were suggested as forming the basis for improved spatial acuity in skin treated with a hydrating product seen in Leveque $e t$ al. Schematic adapted from Figure 7 in Leveque et al. Dotted lines show the extent of skin regions undergoing deflection.

stimulus. Specifically, it is not the case that moisturization would be expected to increase sensitivity to all stimuli, instead we suggest two hypotheses:

- Fixed-force hypothesis: A fixed-force stimulus would lead to greater depth of indentation in more compliant skin, and thus potentially greater mechanoreceptor activity beneath the probe, but less extensive activity in the skin's mechanoreceptors in the neighborhood of the probe. A typical fixed-force stimulus is a Von Frey hair, or - approximately - a two-point stimulator. In this case one would expect greater sensitivity to suprathreshold spatial stimulation, but potentially reduced sensitivity to nonspatial tasks in more compliant skin. For example, the spatial task of Leveque and colleagues supports the former, whereas the latter would be supported by reduced sensitivity to the detection of light touch (e.g., via Von Frey hairs).

- Fixed-depth hypothesis: In contrast, a fixed-depth stimulus would lead to the same activity beneath the probe regardless of skin compliance, and less extensive activity in the skin's mechanoreceptors in the neighborhood of the probe in more compliant skin. Vibrotactile stimuli are often delivered at fixed indentation depth [83]. In this case one might anticipate greater sensitivity to fixed-depth stimuli when the skin is less compliant (e.g., less hydrated). However, there are two competing mechanisms at play here, namely locally great but non-extensive activity versus locally small but more extensive activity. The relative importance of these two factors would determine sensitivity to fixed-depth stimulation.

In ongoing, as yet unpublished experiments, we have collected data for a variety of measures of sensory function, which pertain to these hypotheses. Skin sensory function has been measured with and without hydrating skin treatments. Data for the two creams tested to date replicate the spatial acuity findings of Lévêque et al., but in a younger cohort, tested at the forearm. Additional tests of sensory function suggest changes consistent with increases in skin stiffness. For example, we find that neither of the products tested to date alter detection thresholds to fixed-depth, low frequency $(33 \mathrm{~Hz})$ vibrations, whereas some treatments decrease thresholds to high frequency $(100 \mathrm{~Hz})$ vibrations. Spatial summation is typically not found for the detection of vibration frequencies below $40 \mathrm{~Hz}$, but is found at higher stimulus frequencies [83]. This hints that the threshold changes for the $100 \mathrm{~Hz}$ stimulus occur via a greater extent of skin stimulation, which could occur if the treatment decreased the compliance of the skin (i.e., increased its stiffness). If reduced compliance is seen, this cannot be on the basis of increased skin hydration. Instead, it is possible that the treatment acts as a stiff sheet over the skin. Data collection continues, but these initial results suggest a promising role for quantitative sensory testing beyond tests of spatial acuity while keeping in mind the different expected effects of fixed-force versus fixed-depth stimulation.

Hydration has also been suggested as the basis of differences in the perception of topically applied waters that varied in mineral content [5]. Specifically, it was proposed that greater and more prolonged softness, suppleness and comfort ratings for low mineral content waters occurred because such waters evaporated from the skin more slowly than more mineralized waters. That is, low mineral content was associated with prolonging skin hydration. However, it is also possible that the different waters altered the tribological properties of the skin, which was not explicitly tested.

In summary it is clear that skin care products can influence the skin's mechanical properties and thus measured sensory function. This suggests a potential functional benefit of skin care products applied to the skin of older individuals, namely that of alleviating the welldocumented sensory losses that occur with aging [83-87]. Note that skin mechanics do not fully account for the losses in sensory function with aging [88]; innervation 
changes have been reported with advancing age [89]. Central mechanisms are likely to account for some agerelated declines, too. Additionally, nociception (i.e., the perception of painful stimuli), unlike the perception of innocuous stimuli, does not appear to be dependent on skin mechanics [90], perhaps because of the relatively strong (e.g., high magnitude) nature of nociceptive stimuli, and therefore is unlikely to be influenced by skin care products.

\section{Conclusions}

A problem with many of the associations reported here between physical attributes of skin care products and percepts is that these have typically taken a single or very limited set of physical parameters and related them to many perceptual attributes. Relationships are often found using this approach, but it is not clear that the physical parameter is actually that driving perception. For example, viscosity-related parameters are related to many distinct percepts; these percepts, if truly different, cannot be driven by the same underlying physical mechanism. Instead, there must exists unmeasured physical parameters that are themselves correlated with viscosity, or variations with viscosity over time and/or touch behavior lead to different percepts.

Future studies involving force plate devices would seem to be a useful next step in defining physical-perceptual links, for these kinds of studies directly obtain the tribological and mechanical events that occur during actual touch, as opposed to using instrumental measures (rheological and tribological) which may not well represent the complex physical system represented by a finger moving against, and assessing, a lubricated surface. A further advantage of such approaches is that temporal changes in the nature of the mechanical stimulus can be readily gathered, and these are likely to be the basis on which many products are judged perceptually distinct.

\section{REFERENCES}

[1] S. A. Wissing and R. H. Müller, "The Influence of Solid Lipid Nanoparticles on Skin Hydration and Viscoelasticity-in Vivo Study," European Journal of Pharmaceutics and Biopharmaceutics, Vol. 56, No. 1, 2003, pp. 67-72. doi:10.1016/S0939-6411(03)00040-7

[2] J. T. Mortenson, P. Bjerring and M. Cramers, "Locobase Repair (R) Cream Following $\mathrm{CO}_{2}$ Laser Skin Resurfacing Reduces Interstitial Fluid Oozing," Journal of Cosmetic and Laser Therapy, Vol. 3, 2001, pp. 155-158. doi:10.1080/147641701753414979

[3] J. Armendariz-Borunda, et al., "A Controlled Clinical Trial with Pirfenidone in the Treatment of Pathological Skin Scarring Caused by Burns in Pediatric Patients," Annals of Plastic Surgery, Vol. 68, No. 1, 2012, pp. 22-28. doi:10.1097/SAP.0b013e31821b6d08
[4] A. Vararesou, et al., "Efficacy and Tolerance Study of an Oligopeptide with Potential Anti-Aging Activity," Journal of Cosmetics, Dermatological Sciences and Applications, Vol. 1, No. 4, 2011, pp. 133-140. doi:10.4236/jedsa.2011.14020

[5] I. Bacle, et al., "Sensory Analysis of Four Medical Spa Spring Waters Containing Various Mineral Concentrations," International Journal of Dermatology, Vol. 38, No. 10, 1999, pp. 784-786. doi:10.1046/j.1365-4362.1999.00789.x

[6] G. A. Gescheider, S. J. Bolanowski and R. T. Verrillo, "Some Characteristics of Tactile Channels," Behavioral Brain Research, Vol. 148, No. 1, 2004, pp. 35-40. doi:10.1016/S0166-4328(03)00177-3

[7] N. Domoto, et al., "Evaluation of the Efficacy of Orange roughy (Hoplostetbus atlanticus) Oil in Subjects with Dry skin," International Journal of Cosmetic Science, Vol. 34, No. 4, 2012, pp. 322-327. doi:10.1111/j.1468-2494.2012.00719.x

[8] M. Zampini, S. Guest and C. Spence, "The Role of Auditory Cues in Modulating the Perception of Electric Toothbrushes," Journal of Dental Research, Vol. 82, No. 11, 2003, pp. 929-932. doi:10.1177/154405910308201116

[9] C. Spence and A. Gallace, "Multisensory Design: Reaching Out to Touch the Consumer," Psychology \& Marketing, Vol. 28, No. 3, 2011, pp. 267-308. doi:10.1002/mar.20392

[10] S. Guest, et al., "Sensory and Affective Judgments of Skin during Inter- and Intrapersonal Touch," Acta Psychologica, Vol. 130, No. 2, 2009, pp. 115-126. doi:10.1016/j.actpsy.2008.10.007

[11] M. E. Parente, A. Gámbaro and G. Solana, "Study of Sensory Properties of Emollients Used in Cosmetics and Their Correlation with Physicochemical Properties," Journal of Cosmetic Science, Vol. 56, No. 3, 2005, pp. 175-182.

[12] P. Stern, H. Valentová and J. Pokorný, "Relations between Rheological and Sensory Characteristics of Cosmetic Emulsions," SÖFW Journal, Vol. 123, 1997, pp. 445-448.

[13] M. Hollins, et al., "Perceptual Dimensions of Tactile Surface Texture: A Multidimensional Scaling Analysis," Perception \& Psychophysics, Vol. 54, No. 6, 1993, pp. $697-$ 705. doi:10.3758/BF03211795

[14] M. Hollins, et al., "Individual Differences in Perceptual Space for Tactile Textures: Evidence from Multidimensional Scaling," Perception \& Psychophysics, Vol. 62, No. 8, 2000, pp. 1534-1544. doi:10.3758/BF03212154

[15] D. Picard, et al., "Perceptual Dimensions of Tactile Textures," Acta Psychologica, Vol. 114, No. 2, 2003, pp. 165184. doi:10.1016/j.actpsy.2003.08.001

[16] W. M. Bergmann Tiest and A. M. I. Kappers, "Analysis of Haptic Perception of Materials by Multidimensional Scaling and Physical Measurements of Roughness and Compressibility," Acta Psychologica, Vol. 121, No. 1, 2006, pp. 1-20. doi:10.1016/j.actpsy.2005.04.005

[17] Y. Na and C. Kim, "Quantifying the Handle and Sensibility of Woven Silk Fabrics," Textile Research Journal, Vol. 71, No. 9, 2001, pp. 739-742. doi: $10.1177 / 004051750107100814$

[18] I. F. Almeida, A. R. Gaio and M. F. Bahia, "Skinfeel 
Analysis of Oleogels," Journal of Sensory Studies, Vol. 23, No. 1, 2008, pp. 92-113. doi:10.1111/j.1745-459X.2007.00144.x

[19] I.-S. Lee, et al., "Terminology Development and Panel Training for Sensory Evaluation of Skin Care Products Including Aqua Cream," Journal of Sensory Studies, Vol. 20, No. 5, 2005, pp. 421-433. doi:10.1111/j.1745-459X.2005.00037.x

[20] S. Guest, et al., "The Development and Validation of Sensory and Emotional Scales of Touch Perception," Attention, Perception \& Psychophysics, Vol. 73, No. 2, 2011, pp. 531-550. doi:10.3758/s13414-010-0037-y

[21] S. Guest, et al., "Perception of Fluids with Diverse Rheology Applied to the Axillary versus Volar Forearm Skin," Somatosensory and Motor Research, Vol. 29, 2012, pp. 89-102. doi:10.3109/08990220.2012.686937

[22] R. Melzack, "The McGill Pain Questionnaire: Major Properties and Scoring Methods," Pain, Vol. 1, 1975, pp. 277288. doi:10.1016/0304-3959(75)90044-5

[23] N. Bhushan, A. R. Rao and G. L. Lohse, "The Texture Lexicon: Understanding the Categorization of Visual Texture Terms and Their Relationship to Texture Images," Cognitive Science, Vol. 21, No. 2, 1997, pp. 219-246. doi:10.1207/s15516709 $\operatorname{cog} 2102 \_4$

[24] F. P. McGlone, et al., "Discriminative Touch and Emotional Touch," Canadian Journal of Experimental Psychology, Vol. 61, No. 3, 2007, pp. 175-183. doi: $10.1037 /$ cjep2007019

[25] Å. Vallbo, H. Olausson and J. Wessberg, "Unmyelinated Afferents Constitute a Second System Coding Tactile Stimuli of the Human Hairy Skin," Journal of Neurophysiology, Vol. 81, No. 6, 1999, pp. 2753-2763.

[26] A. Vallbo, et al., "A system of Unmyelinated Afferents for Innocuous Mechanoreception in the Human Skin," Brain Research, Vol. 628, 1993, pp. 301-304. doi:10.1016/0006-8993(93)90968-S

[27] C. E. Osgood, "The Nature and Measurement of Meaning," Psychological Bulletin, Vol. 49, No. 3, 1952, pp. 197-237. doi:10.1037/h0055737

[28] C. E. Osgood and G. J. Suci, "Factor Analysis of Meaning," Journal of Experimental Psychology, Vol. 50, No. 5, 1955, pp. 325-338. doi:10.1037/h0043965

[29] J. A. Russell and A. Mehrabian, "Evidence for a ThreeFactor Theory of Emotions," Journal of Research in Personality, Vol. 11, No. 3, 1977, pp. 273-294. doi:10.1016/0092-6566(77)90037-X

[30] J. A. Russell and J. H. Steiger, "The Structure in Persons' Implicit Taxonomy of Emotions," Journal of Research in Personality, Vol. 16, 1982, pp. 447-469. doi:10.1016/0092-6566(82)90005-8

[31] C. E. Osgood, "Dimensionality of the Semantic Space for Communication via Facial Expressions," Scandinavian Journal of Psychology, Vol. 7, No. 1, 1966, pp. 1-30. doi:10.1111/j.1467-9450.1966.tb01334.x

[32] A. Mehrabian, "A Semantic Space for Nonverbal Behavior," Journal of Consulting and Clinical Psychology, Vol. 35, No. 2, 1970, pp. 248-257. doi:10.1037/h0030083

[33] F. P. McGlone, et al., "Differences in Pleasant Touch
Processing between Glabrous and Hairy Skin in $\mathrm{Hu}-$ mans," European Journal of Neuroscience, Vol. 35, No. 11, 2012, pp. 1782-1788. doi:10.1111/j.1460-9568.2012.08092.x

[34] F. Shama and P. Sherman, "Variation in Stimuli Associated with Oral Evaluation of the Viscosities of Glucose Solutions," Journal of Texture Studies, Vol. 4, No. 2, 1973, pp. 254-262. doi:10.1111/j.1745-4603.1973.tb00669.x

[35] F. Shama and P. Sherman, "Identiciation of Stimuli Controlling the Sensory Evaluation of Viscosity II, Oral Methods." Journal of Texture Studies, Vol. 4, No. 1, 1973, pp. 111-118. doi:10.1111/j.1745-4603.1973.tb00657.x

[36] F. W. Wood, "Psychophysical Studies on the Consistency of Liquid Foods," Rheology and Texture of Foodstuffs, SCI Monograph No. 27, London, 1968, pp. 40-49.

[37] A. K. Smith, H. June and A. C. Noble, "Effects of Viscosity on the Bitterness and Astringency of Grape Seed Tannin," Food Quality and Preference, Vol. 7, No. 3, 1996, pp. 161-166. doi:10.1016/S0950-3293(96)00028-6

[38] L. Engelen, et al., "Oral Size Perception of Particles: Effect of Size, Type, Viscosity and Method," Journal of Texture Studies, Vol. 36, No. 4, 2005, pp. 373-386. doi:10.1111/j.1745-4603.2005.00022.x

[39] M. R. Wegener, "A Psycho-Rheological Study of SkinFeel," University of Bristol, Bristol, 1997.

[40] S. S. Stevens and M. Guirao, "Scaling of Apparent Viscosity," Science, Vol. 144, No. 3622, 1964, pp. 1157-1158. doi:10.1126/science.144.3622.1157

[41] F. Shama, C. Parkinson and P. Sherman, "Identification of Stimuli Controlling the Sensory Evaluation of Viscosity I. Non-Oral Methods," Journal of Texture Studies, Vol. 4, No. 1, 1973, pp. 102-110. doi:10.1111/j.1745-4603.1973.tb00656.x

[42] R. Brummer and S. Godersky, "Rheological Studies to Objectify Sensations Occurring when Cosmetic Emulsions Are Applied to the Skin," Colloids and Surfaces A: Physicochemical and Engineering Aspects, Vol. 152, No. 1-2, 1999, pp. 89-94. doi:10.1016/S0927-7757(98)00626-8

[43] R. H. Jellema, et al., "Relating the Sensory Sensation 'Creamy Mouthfeel' in Custards to Rheological Measurements," Journal of Chemometrics, Vol. 19, No. 3, 2005, pp. 191-200. doi:10.1002/cem.922

[44] R. E. Greenaway, "Psychorheology of Skin Cream," University of Nottingham, Nottingham, 2010.

[45] M. J. Adams, et al., "Finger Pad Friction and Its Role in Grip and Touch," Journal of the Royal Society Interface, Vol. 10, No. 80, 2013, Article ID: 20120467.

[46] S. Derler and L.-C. Gerhardt, "Tribology of Skin: Review and Analysis of Experimental Results for the Friction Coefficient of Human Skin," Tribology Letters, Vol. 45, No. 1, 2012, pp. 1-27. doi:10.1007/s11249-011-9854-y

[47] L.-C. Gerhardt, et al., "Study of Skin-Fabric Interactions of Relevance to Decubitus: Friction and Contact-Pressure Measurements," Skin Research and Technology, Vol. 14, No. 1, 2008, pp. 77-88.

[48] S. M. Pasumarty, et al., "Friction of the Human Finger Pad: Influence of Moisture, Occlusion and Velocity," Tri- 
bology Letters, Vol. 44, No. 2, 2011, pp. 117-137. doi:10.1007/s11249-011-9828-0

[49] A. B. Cua, K. P. Wilhelm and H. I. Maibach, "Frictional Properties of Human Skin: Relation to Age, Sex and Anatomical Region, Stratum Corneum Hydration and Transepidermal Water Loss," British Journal of Dermatology, Vol. 123, No. 4, 1990, pp. 473-479. doi:10.1111/j.1365-2133.1990.tb01452.x

[50] S. Nacht, et al., "Skin Friction Coefficient: Changes Induced by Skin Hydration and Emollient Application and Correlation with Perceived Skin Feel," Journal of the Society of Cosmetic Chemists, Vol. 32, No. 2, 1981, pp. 5565.

[51] J. Chen and J. R. Stokes, "Rheology and Tribology: Two Distinctive Regimes of Food Texture Sensation," Trends in Food Science \& Technology, Vol. 25, No. 1, 2012, pp. 4-12. doi:10.1016/j.tifs.2011.11.006

[52] J. H. H. Bongaerts, K. Fourtouni and J. R. Stokes, "SoftTribology: Lubrication in a Compliant PDMS-PDMS Contact," Tribology International, Vol. 40, 2007, pp. 15311542. doi:10.1016/j.triboint.2007.01.007

[53] J. R. Stokes, et al., "Lubrication, Adsorption, and Rheology of Aqueous Polysaccharide Solution," Langmuir, Vol. 27, No. 7, 2011, pp. 3474-3484. doi:10.1021/la104040d

[54] A. M. Smith, G. Gosselin and B. Houde, "Deployment of Fingertip Forces in Tactile Exploration," Experimental Brain Research, Vol. 147, No. 2, 2002, pp. 209-218. doi:10.1007/s00221-002-1240-4

[55] G. K. Essick, et al., "Quantitative Assessment of Pleasant Touch," Neuroscience and Biobehavioral Reviews, Vol. 34, No. 2, 2010, pp. 192-203. doi:10.1016/j.neubiorev.2009.02.003

[56] B. Bhushan, G. Wei and P. Haddad, "Friction and Wear Studies of Human Hair And Skin," Wear, Vol. 258, No. 9, 2005, pp. 1012-1021. doi:10.1016/j.wear.2004.12.026

[57] A. Dussaud, J. Ding and A. Lips, "Method and System for Characterizing Tactile Perception," 2005.

[58] A. M. Smith, et al., "Role of Friction and Tangential Force Variation in the Subjective Scaling of Tactile Roughness," Experimental Brain Research, Vol. 144, 2002, pp. 211-223. doi:10.1007/s00221-002-1015-y

[59] M. G. Gee, et al., "A New Friction Measurement System for the Frictional Component of Touch," Wear, Vol. 259, No. 2, 2005, pp. 1437-1442. doi:10.1016/j.wear.2005.02.053

[60] L. Skedung, et al., "Finger Friction Measurements on Coated and Uncoated Printing Papers," Tribology Letters, Vol. 37, No. 2, 2010, pp. 389-399. doi:10.1007/s11249-009-9538-z

[61] S. Guest, et al., "Physics and Tactile Perception of FluidCovered Surfaces," Journal of Texture Studies, Vol. 43, No. 1, 2012, pp. 77-93. doi:10.1111/j.1745-4603.2011.00318.x

[62] K. Nakano, et al., "Tribological Method to Objectify Similarity of Vague Tactile Sensations Experienced during Application of Liquid Cosmetic Foundations," Tribology International, in press.

[63] K. Horiuchi, et al., "Relationship between Tactile Sensa- tion and Friction Signals in Cosmetic Foundation," Tribology Letters, Vol. 36, No. 2, 2009, pp. 113-123. doi:10.1007/s11249-009-9466-y

[64] K. Nakano, et al., "A Neural Network Approach to Predict Tactile Comfort of Applying Cosmetic Foundation," Tribology International, Vol. 43, No. 11, 2010, pp. 19781990. doi:10.1016/j.triboint.2010.04.004

[65] F. Shao, T. H. C. Childs and B. Henson, "Developing an Artificial Fingertip with Human Friction Properties," Tribology International, Vol. 42, No. 11-12, 2009, pp. 15751581. doi:10.1016/j.triboint.2009.02.005

[66] C. M. Oddo, et al., "Roughness Encoding for Discrimination of Surfaces in Artificial Active-Touch," IEEE Transactions on Robotics, Vol. 27, No. 3, 2011, pp. 522-533. doi:10.1109/TRO.2011.2116930

[67] G. J. van den Oever, "Integrated Sensory Response (ISR) Modeling: A New Methodology to Understand and Predict Sensory Attributes in Terms of Physical Properties," Cereal Chemistry, Vol. 80, No. 4, 2003, pp. 409-418. doi:10.1094/CCHEM.2003.80.4.409

[68] G. J. Van den Oever, et al., "Design of Foods for the Optimal Delivery of Basic Tastes," In: D. J. McClements and E. Decker, Eds., Designing Functional Foods: Measuring and Controlling Food Structure Breakdown and Nutrient Absorption, University of Massachusetts, Massachusetts, 2009.

[69] R. Ackerley, et al., "Wetness Perception across Body Sites," Neuroscience Letters, Vol. 522, No. 1, 2012, pp. 73-77. doi:10.1016/j.neulet.2012.06.020

[70] W. M. Bergmann Tiest, et al., "Haptic Perception of Wetness," Acta Psychologica, Vol. 141, No. 2, 2012, pp. 159163. doi:10.1016/j.actpsy.2012.07.014

[71] I. Bershansky, "Thunberg's Illusion," American Journal of Psychology, Vol. 34, No. 2, 1923, pp. 291-295. doi: $10.2307 / 1413584$

[72] I. M. Bentley, "The Synthetic Experiment," American Journal of Psychology, Vol. 11, 1900, pp. 405-426. doi: $10.2307 / 1412750$

[73] C. E. Lauterbach and R. E. Crouser, "Sensation Cues to Moisture," Journal of Experimental Psychology, Vol. 16, No. 2, 1933, pp. 323-338. doi:10.1037/h0073310

[74] S. Guest, et al., "Oral Hydration, Parotid Salivation and the Perceived Pleasantness of Small Water Volumes," Physiology \& Behavior, Vol. 89, No. 5, 2006, pp. 724734. doi:10.1016/j.physbeh.2006.08.012

[75] E. Lehmuskallio and H. Anttonen, "Thermophysical Effects of Ointments in Cold: An Experimental Study with a Skin Model," Acta Dermato-Venereologica, Vol. 79, No. 1, 1999, pp. 33-36. doi:10.1080/000155599750011660

[76] E. Lehmuskallio, "Cold Protecting Emollients and Frostbite," University of Oulu, Oulu, 2001, p. 96.

[77] Y. Nonomura, et al., "Tactile Impression and Friction of Water on Human Skin," Colloids and Surfaces B: Biointerfaces, Vol. 69, No. 2, 2009, pp. 264-267. doi:10.1016/j.colsurfb.2008.11.024

[78] M. J. Adams, B. J. Briscoe and S. A. Johnson, "Friction and Lubrication of Human Skin," Tribology Letters, Vol. 26, No. 3, 2007, pp. 239-253. 


$$
\text { doi:10.1007/s11249-007-9206-0 }
$$

[79] J. de Rigal and J.-L. Lévêque, "In Vivo Measurement of the Stratum Corneum Elasticity," Bioengineering and the Skin, Vol. 1, No. 1, 1985, pp. 13-23.

[80] F. M. Hendricks, et al., "Influence of Hydration and Experimental Length Scale on the Mechanical Response of Human Skin in Vivo, Using Optical Coherence Tomography," Skin Research and Technology, Vol. 10, No. 1, 2004, pp. 231-241. doi:10.1111/j.1600-0846.2004.00077.x

[81] E. S. Dellon, et al., "The Relationships between Skin Hardness, Pressure Perception and Two-Point Discrimination in the Fingertip," Journal of Hand Surgery. British Volume, Vol. 20, No. 1, 1995, pp. 44-48. doi:10.1016/S0266-7681(05)80015-4

[82] J.-L. Lévêque, et al., "Changes in Tactile Spatial Discrimination and Cutaneous Coding Properties by Skin Hydration in the Elderly," Journal of Investigative Dermatology, Vol. 115, No. 3, 2000, pp. 454-458. doi:10.1046/j.1523-1747.2000.00055.x

[83] G. A. Gescheider, et al., "The Effects of Aging on Information-Processing Channels in the Sense of Touch: I. Absolute Sensitivity," Somatosensory and Motor Research, Vol. 11, 1994, pp. 345-357. doi: $10.3109 / 08990229409028878$

[84] J. C. Stevens and K. K. Choo, "Spatial Acuity of the Body Surface over the Life Span," Somatosensory and Motor Research, Vol. 13, No. 2, 1996, pp. 153-166. doi: $10.3109 / 08990229609051403$

[85] J. C. Stevens and K. K. Choo, "Temperature Sensitivity of the Body Surface over the Life Span," Somatosensory and Motor Research, Vol. 15, No. 1, 1998, pp. 13-28. doi:10.1080/08990229870925

[86] G. A. Gescheider, et al., "The Effects of Aging on Information-Processing Channels in the Sense of Touch: III. Differential Sensitivity to Changes in Stimulus Intensity," Somatosensory and Motor Research, Vol. 13, 1996, pp. 73-80. doi:10.3109/08990229609028914

[87] M. Stuart, et al., "Effects of Aging on Vibration Detection Thresholds at Various Body Regions," BMC Geriatrics, Vol. 3, No. 1, 2003, p. 1. doi:10.1186/1471-2318-3-1

[88] K. L. Woodward, "The Relationship between Skin Compliance, Age, Gender, and Tactile Discriminative Thresholds in Humans," Somatosensory and Motor Research, Vol. 10, No. 1, 1993, pp. 63-67. doi: $10.3109 / 08990229309028824$

[89] I. Besné, C. Descombes and L. Breton, "Effect of Age and Anatomical Site on Density of Sensory Innervation Inc. Nerve Endings in Human Epidermis," Archives of Dermatology, Vol. 138, No. 11, 2002, pp. 1445- 1450. doi:10.1001/archderm.138.11.1445

[90] J. D. Greenspan and S. L. B. McGillis, "Stimulus Feastures Relevant to the Perception of Sharpness and Mechanically Evoked Cutaneous Pain," Somatosensory and Motor Research, Vol. 8, No. 2, 1991, pp. 137-147. doi:10.3109/08990229109144738 